\title{
Reply: A unified classification system for eukaryotic transposable elements should reflect their phylogeny
}

\author{
Thomas Wicker, François Sabot, Aurélie Hua-Van, Jeffrey L. Bennetzen, \\ Pierre Capy, Boulos Chalhoub, Andrew Flavell, Philippe Leroy, \\ Michele Morgante, Olivier Panaud, Etienne Paux, Phillip SanMiguel and \\ Alan H. Schulman
}

The goal of our Guidelines article (A unified classification system for eukaryotic transposable elements Nature Rev. Genet. 8, 973-982 (2007) $)^{1}$, as Seberg and Petersen correctly surmise (A unified classification system for eukaryotic transposable elements should reflect their phylogeny. Nature Rev. Genet. 24 Feb 2009 (doi:10.1038/ $\operatorname{nrg} 2165-\mathrm{c} 1))^{2}$, was to lay out a system of nomenclature and classification that assists annotators and lays the groundwork for future functional analyses. In pursuing this task, we followed the lead of bacteriologists ${ }^{3}$ and virologists ${ }^{4}$. Seberg and Petersen raise two main points: first they address our phylogenetic system (with which they do not take issue), and second they criticize the rules used to achieve monophyletic groupings. They argue against the $80-80-80$ rule, but fail to propose a practical alternative. Our experience tells us that $80-80-80$ works reasonably well and we propose it as a pragmatic solution.

Seberg and Petersen also bring us to several deeper issues. First, to what extent do genetic elements such as viruses and transposable elements (TEs) mirror organisms for which phylogenetic schemes have been devised? Both viruses and TEs can recombine in ways that are rare in most organisms, producing polyphyly and networks rather than easily discernible phylogenies. Second, the sizes and structures of TE populations are very different from those of organisms. TEs mutate during propagation at rates up to $10^{3}$-fold higher than that of nuclear genes or of integrated TE copies ${ }^{5}$. A single cell may easily contain 5,000 copies of a particular TE, and a leaf at least 3,000 cells ${ }^{6}$. As Seberg and Petersen note, TEs do not solely follow the phylogeny of their host organisms; they were divided into multiple groups of many members even before the divergence of the main branches of the eukaryotes and may be subject as well to horizontal transfer. The situation is very different for genes that replicate only as part of the nuclear genome - these are much less repetitious, and diverge at much lower rates.

We would also like to point out that taxonomy is a work in progress, and has relied upon a variety of approaches. Organismal taxonomists achieved much by classifying species on the basis of visible characteristics derived from the expression of many genes (for example, floral morphology), rather than their nucleic acid sequences. Organismal taxonomies are often polyphyletic, leading to later division. Taxonomists, in turn, are often divided into 'lumpers' and 'splitters' depending on their personal predilections; our annotation system leaves room for both. The concept of autonomous and non-autonomous members of a TE clade is foreign to organismal taxonomy. Many non-autonomous TEs are derived from and are parasitic on autonomous TEs, whereas others may be parasitic on non-related TEs. For the latter, it will be important to define their synapomorphies (that is, derived characteristics shared by several taxa), but the functional relationships must first be discovered.

In summary, we wish to emphasize that the problem of classification is very different for extranuclear genetic elements than for organisms. What is needed, given the rapidity of sequencing and the size of annotation tasks, are clear rules that work most of the time and a system that accommodates both current uncertainties and future discoveries.

Published online 24 February 2009

1. Wicker, T. et al. A unified classification system for eukaryotic transposable elements. Nature Rev. Genet. 8, 973-982 (2007).

2. Seberg, O. \& Petersen, G. A unified classification system for eukaryotic transposable elements should reflect their phylogeny. Nature Rev. Genet. 24 Feb 2009 (doi: 10.1038/nrg2165-c3).

3. Garrity, G. M. et al. Taxonomic Outline of the Bacteria and Archaea (Michigan State University Board of Trustees) [online], < http://www. taxonomicoutline.org/index.php/toba/article/ view/190/223 > (2007)

4. Fauquet, C. M., Mayo, M. A., Maniloff, J., Desselberger, U. \& Ball, L. A. (eds) Virus Taxonomy: VIIIth Report of the International Committee on Taxonomy of Viruses (Academic Press, Amsterdam, The Netherlands, 2005).

5. Gabriel, A., Willems, M., Mules, E. H. \& Boeke, J. D. Replication infidelity during a single cycle of Ty 1 retrotransposition. Proc. Natl Acad. Sci. USA 93, 7767-7771 (1996).

6. Autran, D. et al. Cell numbers and leaf development in Arabidopsis: a functional analysis of the STRUWWELPETER gene. EMBO J. 22, 6036-6049 (2002). 